Department of Paediatric Surgery, Alder Hey Children's Hospital NHS Foundation Trust, Liverpool, UK

2 Institute of Systems and Molecular Biology, University of Liverpool, Liverpool, UK

3 Institute of Life Course and Medical Sciences, University of Liverpool, Liverpool, UK

Cite this as: $B M J 2021 ; 375: n 2785$ http://dx.doi.org/10.1136/bmj.n2785 Published: 12 November 2021

\section{Training and the future delivery of UK paediatric surgery in the NHS}

\author{
Rachel Harwood, ${ }^{1,2}$ Paul D Losty ${ }^{3}$
}

Paediatric surgery is a highly specialised surgical field requiring expertise in caring for children with common and rare conditions. The creation of $26 \mathrm{UK}$ paediatric surgery centres has occurred over recent decades, resulting in a geographical disparity, both in location and number of centres. ${ }^{1}$ Accumulation of experience and advances in medical technologies has resulted in a huge success story for the specialty. Many infants and children, hitherto with severe or potentially fatal conditions, now survive and thrive. General surgeons regularly undertake common paediatric elective and emergency procedures, although nationally there now is a steady drift of these operations being undertaken by specialty paediatric surgeons. Furthermore individual paediatric surgeon specific index case-numbers of some complex rare conditions has led in some instances to the transfer of healthcare to other specialist trusts for multi-disciplinary input and bespoke services.

NHS England and Improvement commissioned a Getting it Right First Time (GIRFT) report into paediatric surgery provision in England in 2018 and its early findings were supported (in principle) by the British Association of Paediatric Surgeons. ${ }^{2}$ The report describes the crucial need for concentration of sub-specialist surgical services in fewer (nominally 10) institutions in England for the expert care of children with varied index rare conditions.

Sub-specialist centres are expected to have a "critical mass" of sub-specialist paediatric upper and lower gastro-intestinal and urology surgeons in addition to specialist paediatric surgeons in other

sub-disciplines e.g. surgical oncology. It is anticipated that other paediatric surgical units would continue to care for infants and children in their immediate geographic locality and community who do not have the index rare conditions, many of whom will require care that cannot be provided by a non-specialist team.

A specialty service where this is already well practised is paediatric cardiac surgery and studies clearly show improvement in outcome metrics with a higher volume of complex index cases. ${ }^{3}$ Similarly, concentration of care of infants with biliary atresia and bladder exstrophy has led to marked noteworthy improvements in overall outcomes. ${ }^{45}$ While the effects of "super centralisation" on patient clinical outcomes appears perhaps self evident, the "knock on" effects on paediatric surgery specialty training are likely to be very significant. Paediatric surgical training is generically themed and wide-ranging, with surgeons completing their training with a limited ability to immediately sub-specialise. Some trainees will be enthusiastically driven to undertake a sub-specialty fellowship after certification of completion of training (CCT), but the majority currently take up a consultant post without bespoke sub-specialist training.

Centralisation of sub-specialist care for children with rare index surgical disorders gives opportunity to deliver sub-specialist training.

Over the past two decades UK surgical training has changed immeasurably. A reduction in hours due to the European Working Time Directive and a significant diminution in the number of years required to achieve CCT has resulted in fewer operating hours gained and less non-operative clinical experience before consultancy. ${ }^{6}$ Sweeping changes to the delivery of non-specialist children's surgical care by adult general surgeons is likely in the wake of the covid-19 pandemic. This may result in a shift of "low-volume" specialist paediatric centres undertaking more "low complexity" elective childhood surgery which has been unduly delayed, with growing waiting times in secondary hospitals, potentially further reducing the experience of the adult general surgeon in delivering "general surgery of childhood."

There are a range of approaches to be tactfully considered to better provide effective specialist and sub-specialist training for paediatric surgeons. The first option, cited within the GIRFT report, follows ideologically and thematically the American surgical training model where a five year general surgery residency is undertaken with licence accreditation and then later competitive nationwide entry into a two year paediatric surgery fellowship programme. ${ }^{7}$ This system resourcefully promotes "the pathway in surgical training" as it crucially allows the resident to grow operative skills in general surgery with a likely higher volume of minimally invasive surgery index case numbers and gives opportunities to learn "cutting edge" innovative techniques rarely deployed in paediatric surgery (e.g. robotic surgery).

The second option that is workable is to mandate that trainees wishing to sub-specialise as a consultant paediatric surgeon must undertake a post-CCT clinical fellowship of 12-24 months duration. This would provide great opportunity for surgical fellowship led training at a designated centre (UK or overseas) before individuals seek substantive consultant appointment with recognised sub-specialty credits. If this approach were to be taken, new UK training fellowship posts need to be created and optimised to enable responsive future workforce planning in paediatric surgery. Fellowships should ideally combine clinical and research training potentially with attainment of a higher degree credential to ensure that such surgeons thereafter become sub-specialist consultants.

The third option plausible is a "hybrid" of the above themes. At present, UK medical paediatric trainees wishing to sub-specialise do so by applying to the 
"National Grid System." ${ }^{8}$ A similar format could be effortlessly considered for paediatric surgery with trainees rotated to both specialist and sub-specialist surgical centres within the first four years of a higher specialty training post and then, if so desired, apply for subspeciality clinical fellowship training within their final two years of training. Future work-force planning could be pragmatically managed to ensure that the "critical mass" required number of sub-specialist UK consultant paediatric surgery posts are actively catered for by a system model focused on personalised training. The UK and Ireland Intercollegiate Specialty Board Fellowship Examinations have set a precedent in general surgery in that the oral viva voce section of the FRCS(Gen) exam focuses on testing the exam candidate's knowledge in general surgery and a well defined sub-specialty interest as identified by the examinees. A similar approach could thus be applied to the paediatric surgery examination for surgeon candidates who wish to have credits recognised in sub-specialist training such as urology, oncology, upper and lower tract gastroenterology.

UK paediatric surgical training needs re-configuration as major changes are emerging on the horizon with the new proposed future delivery of children's surgical healthcare in the NHS. It is crucial that the paediatric surgeons in training who will be "tomorrow's surgeons" are wholly confident, competent, and suitably experienced for the specialty role including sub-specialty consultant appointment. Consultation and active dialogue with the surgical Royal Colleges, UK Paediatric Surgical Consultant Workforce, British Association of Paediatric Surgeons (BAPS), allied professional health care organisations, parents, patients, carers and the trainees themselves is key assurance that the new generation of consultant paediatric surgeons will provide the high quality care required for children and families whom they will faithfully serve in the future. ${ }^{9}$

Conflicting interests: PDL served as a Training Programme Director for Paediatric Surgery and a STEC Chair in the Liverpool-Manchester North West England Consortium. PDL served as a University Academic Surgeon Representative on the UK Specialist Advisory Committee (SAC) Paediatric Surgery. PDL is currently an Academic Surgery Representative Interview Panel Member on the UK National Paediatric Surgery (NTN) Training Selection Committee. None further declared.

Provenance and peer review: Not commissioned, not peer reviewed

1 Spitz L. The history of paediatric surgery in the United Kingdom and the influence of the national health service on its development. J Pediatr Surg 2012;47:29-35. doi: 10.1016/j.jpedsurg.2011.10.014. pmid: 22244388

2 BAPS. BAPS Statement of Support for GIRFT National Report on Paediatric Surgery, <https://www.baps.org.uk/news/announcements/baps-statement-of-support-for-girft-nationalreport-on-paediatric-surgery/> (2020).

3 Hannan EL, Racz M, Kavey RE, Quaegebeur JM, Williams R. Pediatric cardiac surgery: the effect of hospital and surgeon volume on in-hospital mortality. Pediatrics 1998;101:963-9. doi: 10.1542/peds.101.6.963. pmid: 9606220

4 Dickson AP. The management of bladder exstrophy: the Manchester experience. J Pediatr Surg 2014;49:244-50. doi: 10.1016/j.jpedsurg.2013.11.031. pmid: 24528959

5 McKiernan PJ, Baker AJ, Kelly DA. The frequency and outcome of biliary atresia in the UK and Ireland. Lancet 2000;355:25-9. doi: 10.1016/S0140-6736(99)03492-3. pmid: 10615887

6 Mowbray N. How to regain those lost training hours. BMJ 2015. doi: 10.1136/bmj.h3405.

7 Fallat ME. Redefining Ladd's path. J Pediatr Surg 2017;52:3-15. doi: 10.1016/j.jpedsurg.2016.10.012. pmid: 27856009

8 RCPCH. Sub-speciality training (NTN grid) - application guidance, 〈https://www.rcpch.ac.uk/resources/sub-specialty-training-ntn-grid-application-guidance> (2021).

9 Losty P. Academic paediatric surgery - Why not?... Semin Pediatr Surg 2021;30:151021. doi: 10.1016/j.sempedsurg.2021.151021. pmid: 33648708 